\title{
Étude de l'expansion du panache plasma formé lors de l'interaction entre un laser ultraviolet et une cible de nitrure de bore ; application à la réalisation de couches minces
}

\author{
B. Angleraud, J. Aubreton et A. Catherinot \\ Laboratoire Matériaux Céramiques et Traitements de Surface, URA 320 du CNRS, \\ Faculté des Sciences, 123 avenue A. Thomas, 87060 Limoges cedex, France
}

\begin{abstract}
Résumé : Consacré à la réalisation de couches minces de nitrure de bore (BN) par ablation laser. le travail présenté consiste d'une part, en l'étude de l'éjection de la matière. provoquée par l'irradiation par un faisceau laser, au-dessus d'une cible de BN et d'autre part, en la réalisation de dépôts de $\mathrm{BN}$ et de l'influence de divers paramètres expérimentaux sur la qualité et les propriétés physico-chimiques des couches obtenues.
\end{abstract}

La cible de BN, placée dans une enceinte à vide, est irradiée par un laser impulsionnel (durée d'impulsion $20 \mathrm{~ns})$ à excimères $(\mathrm{KrF}, \lambda=248 \mathrm{~nm})$. Pénétrant par un hublot de l'enceinte, le faisceau laser est focalisé sur la cible avec une incidence de $45^{\circ}$.

L'expansion du plasma de matière éjectée est suivie par imagerie CCD ultra rapide. La caméra est placée de telle façon que le plan de la matrice CCD soit parallèle à la normale à la cible. Le temps d'exposition la caméra est de $10 \mathrm{~ns}$, et nous avons fait varier le délai $t$ d'observation par rapport au début de l'impulsion laser entre quelques dizaines de ns et quelques $\mu \mathrm{s}$. Ainsi les différentes images obtenues permettent de suivre l'évolution temporelle et spatiale (2D) des espèces émettrices au-dessus de la cible de BN.

En traçant la hauteur $\mathrm{h}$ atteinte par le front du panache lumineux au-dessus de la cible en fonction du temps $\mathrm{t}$ à trois pressions régnant dans l'enceinte (pression résiduelle de $5 \times 10^{-3} \mathrm{~Pa}$, 10 et $500 \mathrm{~Pa}$ en azote), et pour trois fluences laser $\left(7,30\right.$ et $\left.200 \mathrm{~J} / \mathrm{cm}^{2}\right)$, nous pouvons en déduire la vitesse et l'énergie cinétique des espèces éjectées. La vitesse varie de $6,4 \mathrm{~km} / \mathrm{s}$ à 500 $\mathrm{Pa}\left(\dot{\mathrm{a}} 7 \mathrm{~J} / \mathrm{cm}^{2}\right)$ à $90 \mathrm{~km} / \mathrm{s}$ à $5 \times 10^{-3} \mathrm{~Pa}\left(200 \mathrm{~J} / \mathrm{cm}^{2}\right)$. Ceci correspond à des énergies cinétiques variant de 2,7 à $500 \mathrm{eV}$ (masse moyenne des composants éjectés $(B, N): 12,5 \mathrm{~g} / \mathrm{mol}$ )

De plus l'évolution du panache plasma au-dessus de la cible peut être décrite qualitativement à partir de différents modèles théoriques. Un exemple de courbe est représenté sur la figure 1 , à $10 \mathrm{~Pa}$ et $7 \mathrm{~J} / \mathrm{cm}^{2}$.

Sous vide, $\mathrm{h}$ suit une loi linéaire de $t$, le régime d'expansion est libre, le plasma s'étend librement.

A $10 \mathrm{~Pa}$ et $500 \mathrm{~Pa}$, le régime d'expansion change avec la hauteur $\mathrm{h}$ atteinte par le front du panache de matière éjectée au-dessus de la cible :

- pour $\mathrm{h}<2 \mathrm{~cm}$, $\mathrm{h}$ suit une loi linéaire de $\mathrm{t}$, le régime d'expansion est libre;

- pour $1<\mathrm{h}<2-2,5 \mathrm{~cm}$, h suit une loi du type [1]:

$$
\mathrm{h}=\mathrm{t}^{\mathrm{a}} \text { avec } \mathrm{a} \approx 0,4 .
$$

L'expansion peut être décrite par le déplacement d'une onde de choc créé au-dessus du front du plasma;

- pour $\mathrm{h}>3 \mathrm{~cm}$, les espèces ralenties par les collisions avec les molécules d'azote ambiantes, atteignent une hauteur limite $h_{\text {lim }}$ au-dessus de la cible, $h$ suit une loi en exponentielle décroissante de $t$ traduisant un régime de forces de viscosité [2] : 
$(\beta$ : coefficient de freinage)

$$
h=h_{\lim } \cdot\left(1-e^{-\beta \cdot t}\right),
$$

Ces différents régimes se retrouvent lorsque l'on suit le déplacement du centre de masse du panache lumineux (représentant le mouvement de l'ensemble de la matière éjectée) avec des vitesses environ deux fois plus faibles.

A partir de ces résultats, nous avons appliqué la technique de l'ablation par laser UV à la réalisation de dépôt de $\mathrm{BN}$ sur silicium. En assistant cette méthode par un bombardement de la couche en croissance par des ions énergétiques (créés dans un canon à ions), il est possible, dans certaines conditions, d'obtenir des films contenant des liaisons $\mathrm{sp}^{3}$ (caractéristiques du BN de structure cubique, matériau possédant des propriétés comparables à celles du diamant). Notre étude a consisté à déterminer les valeurs des paramètres expérimentaux (fluence, énergie des ions du canon,...) favorisant la création d'un maximum de liaisons $\mathrm{sp}^{3}$.

Il ressort de ce travail que la formation du BN-c est un phénomène à seuil très brutal, caractérisée par des domaines de paramètres expérimentaux très réduits, limités et interdépendants. Par exemple, le pourcentage de liaisons $\mathrm{sp}^{3}$ en fonction de l'énergie des ions du canon est représenté sur la figure 2. $700 \mathrm{eV}$ apparait comme une valeur optimale de l'énergie permettant d'obtenir un pourcentage de liaisons $\mathrm{sp}^{3}$ maximum avec une densité de courant d'ions de $1 \mathrm{~mA} / \mathrm{cm}^{2}$ (bombardement de la couche en croissance par un ion par particule éjectée environ). En optimisant les valeurs des paramètres expérimentaux, les couches réalisées par cette technique ont des pourcentages de liaisons $\mathrm{sp}^{3}$ atteignant $80 \%$ avec un rapport azote/bore d'environ 0,9 ( $\approx$ à celui de la cible).

(Ce travail est partiellement soutenu par la DRET)

\section{Références}

[1] Zel'dovich Ya.B., Raizer Yu, "Physics of shock wave and high-temperature phenomena", Academic Press, New-York and London (1966), 94.

[2] Geohegan D.B., Thin Solid Films 220 (1992), 138.

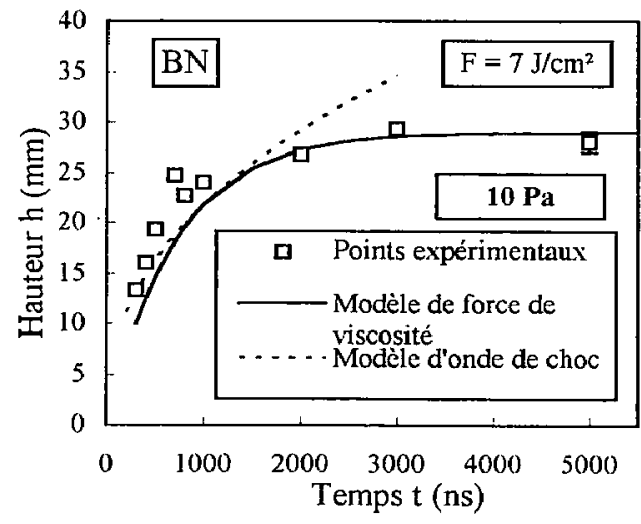

Figure 1 : hauteur atteinte par le front du panache en fonction du temps à $10 \mathrm{~Pa}$.

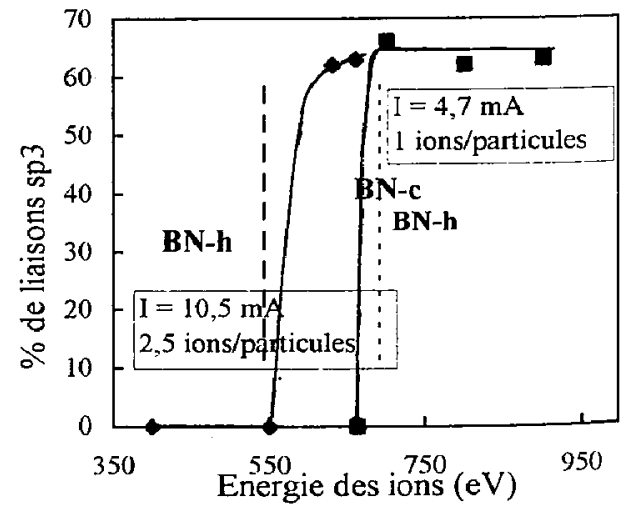

Figure 2 : pourcentage de liaisons $\mathrm{sp}^{3}$ dans les dépôts en fonction de l'énergie des ions. 